\title{
Locating all the Frequency Hopping Components Using Multi-species Particle Swarm Optimization
}

\author{
Guo Jiantao, Wang Lin \\ College of Physics and Electronic Engineering \\ Xinyang Normal University \\ Xinyang, China \\ E:mail: e_jiantao@163.com
}

\begin{abstract}
The particle swarm optimization (PSO) algorithm is applied to the problem of blind parameter estimation of frequency hopping signals. For this target, one Time Frequency representation such as Smoothed Pseudo Wigner-Ville Distribution (SPWVD) is computed firstly. Then, the peaks on TF plane are searched using multispecies PSO. Each particle moves around two dimension time and frequency plane and will converge to different species, which seeds represent the centers of frequency hopping components. A numerical study is carried out for signals which are embedded in a very low SNR ratio noise. Results show that the new method is feasible and much more robust than some existing estimation algorithms.
\end{abstract}

Index Terms-parameter estimation, particle swarm optimization, frequency hopping signals, time frequency representation

\section{INTRODUCTION}

Frequency Hopping (FH) systems have been widely used due to the inherent security features, such as low probability of interception and detection, good capability against interference and hostile jamming in military communications. As the precondition of interception and interference, estimation and tracking the parameters of unknown FH signals are important tasks for information interception and radio sensing with applications in both military and civilian fields. It has become a great challenging problem as the random characteristics of hopping frequency and high levels of noise and interference. To overcome this problem, researches have made many efforts and proposed all kinds of ways for estimating the time and frequency parameters of $\mathrm{FH}$ signals [1]-[3].

Because FH signals are typical non-stationary signals, the frequency domain characteristics at any time of signals are important, so it is essential to describe the joint time frequency (TF) characteristics of FH signals. Research on parameter estimation of FH signals by using time-frequency analysis has been carried out for at least two decades. In these literatures, two philosophies for approaching the parameter estimation problem exist-an adaptive component decomposition approach and kernel design approach. FH signals that consist of various TF structures are represents as a combination of components in the former type, which give important information about inner structures. These components could be obtained by using the matching pursuit algorithm commonly [4]-[6]. But these methods require building parametric, redundant, and well-localized TF atoms, which are matched with FH components difficultly, and high computational time is also a huge bottleneck. In the latter type, FH signals is described generally by a function with time and frequency variables and parameter estimation is operated on the TF plane. Barbarossa [7] proposed a method for estimating hop parameters based on Pseudo Wigner-Ville Distribution (PWVD). There are also cross-terms along frequency axis, but they can often reduced by a two dimensional low-pass filtering of WVD, leading to the smoothed PWVD so that the estimation of hop during parameter is reliable above $4 \mathrm{~dB}$ [8], [9]. However, the SPWVD reduces time and frequency 
resolution while decreasing interference items, where assuming hop during is known in that algorithm. In order to improve the readability and resolution of $\mathrm{TF}$ distribution, Auger and Flandrin proposed a reassignment method [10]. The method reallocates the energy value of arbitrary point in the original TF plane to the center of gravity of these energy contributions, which is applied to parameter estimation by [11]. Image processing techniques are also applied in this problem in [12]. Nevertheless, TF resolution in the above methods is always losing with the reduction of interferences, where the locations of the maximal values of TF distributions appear shift. On the other hand, hop timing can't be correctly estimated using maximum sequence as itself may be wrong and the resulting hop frequency is also a mistake, especially in low SNR noise, although difference operation is simple.

Based on the above analysis, we can see that all the introduced methods must estimate the maximum along time domain or frequency one. This operation is sensitive to noise due to differential operator. In this paper, we propose a new parameter estimation algorithm based on PSO. The proposed estimator utilizes particle search to search peaks in TF plane so as to obtain time and frequency parameter of $\mathrm{FH}$ signals simultaneously. Experiments results illustrate that it can be performed effectively of the proposed algorithm in strong noise environment without any prior knowledge.

This paper is organized as follows. The next section consists of a brief review of PSO and Multi-Species PSO (MSPSO). Then we introduce FH signal model into PSO and give the problem formulation. In section 4 , the new peaks i.e. FH components search algorithm is proposed, whose performance is evaluated and hop during estimation method is also discussed in Section 5. Finally, Section 6 summarizes the conclusions obtained from this research work.

\section{Multi-Species Particle SWARM Optimizer}

Inspiring from the swarms in nature, in 1995, Kennedy, Eberhart and Shi [13] proposed a new heuristic method called PSO based on sociological behavior associated with birds flocking. PSO combines cognition only model that values solely the self-experience and social only model that values solely the experience of neighbors. In particle swarm optimizer, each particle, which represents a potential solution to a problem at hand, adjusts its flying according to its own flying experience and its companions' flying experience. Each particle is treated as a point in a D-dimensional space. The $i^{\text {th }}$ particle is represented as $X_{I}=\left(x_{i 1}, x_{i 1}, \cdots x_{i D}\right)$. The best previous position giving the best fitness value of any particle is recorded and represented as $P_{I}=\left(p_{i 1}, p_{i 1}, \cdots p_{i D}\right)$.The best particle among all the particles in the populations is represented by the symbol g. The velocity for particle $\mathrm{i}$ is represented as $V_{I}=\left(v_{i 1}, v_{i 1}, \cdots v_{i D}\right)$. The particles are manipulated according to the following equation:

$$
\begin{gathered}
v_{i d}(t+1)=w w_{i d}(t)+c_{1} r_{1 d}(t)\left(P_{i d}(t)-x_{i d}(t)\right)+c_{2} r_{2 d}(t)\left(P_{g d}(t)-x_{i d}(t)\right) \\
x_{i d}(t+1)=x_{i d}(t)+v_{i d}(t+1)
\end{gathered}
$$

where $\mathrm{w}$ is the inertia weight factor; $c_{1}$ and $c_{2}$ are cognitive and social acceleration factors, respectively. $c_{1}$ and $c_{2}$ are uniformly random numbers chosen from the interval $[0,1]$. Here, PSO is designed to locate a unique single optimum solution. However, algorithms to find more than one optimum of a function, usually referred to as a multimodal function optimization, have been widely studied since it exists in engineering fields, for example, in this paper [14]-[18]. Previous approaches can be categorized as iterative and subpopulation methods. Parsopoulos and Vrahatis [14] introduced the unified PSO, which requires local and global neighborhoods to be defined aiming their balance. Li proposed [15] the species-based PSO. This method gathers the similar particles into species, which is defined by two variables: species seed and species radius. The particles in the entire swarm move and converge around those seeds, which 
parameters denote the locations of multimodal values. Unfortunately, a cumbersome genetic schedule is necessary to tune the performance of the algorithm [16]. Masao [17], [18] divides the whole population into several subpopulations called species directly. Each species $P_{s}$ occupies a different area of the search space and tries to find out the local minima of that area. Therefore, it can be used to locate all the global minima of the multi-modal function in parallel. If the distance between particle $\mathrm{i}$ and species seed $\mathrm{s}$ at $P_{s}$ is shorter than the threshold $\sigma$ called species radius, then i belongs to species s. In general, the Euclidean distance is used, while it is modified according to the application in this paper and illustrated in the following. Now in MultiSpecies PSO (MSPSO), a global best position $P_{g}$ is replaced by the species best $P_{s}$ that is the best position of the species to which the particle i belongs and (1a) of PSO is replaced by:

$$
v_{i d}(t+1)=w v_{i d}(t)+c_{1} r_{1 d}(t)\left(P_{i d}(t)-X_{i d}(t)\right)+c_{2} r_{2 d}(t)\left(P_{s d}(t)-x_{i d}(t)\right)
$$

\section{SIGNAL MODEL AND PROBLEM FORMULATION}

\section{A. Signal Modal}

The FH signal is modeled as TF shift result of a single tone $x_{0}(t)$, i.e. consists of some components:

$$
x(t)=\sum_{i} X_{i}(t)=\sum_{i} X_{0}\left(t-t_{i}\right) \cos \left(2 \pi f_{i} t+\phi_{i}\right)+n(t) \quad 0<t \leq T
$$

where $x_{0}(t)=\operatorname{rect}_{T_{H}}\left(t-T_{H}-\alpha T_{H}\right) \cos (2 \pi f t), \operatorname{rect}_{T_{h}}(t)$ is equal to one for $t \in\left[-\frac{T_{h}}{2}, \frac{T_{h}}{2}\right]$ and zeros elsewhere. $f_{i}$ is the generic hop frequency, $T_{H}$ is the hop duration, $\alpha T_{H}$ is the hop timing, $\phi_{i}$ is the initial phase of each hop, $n(t)$ is additive noise. $T_{H}, f_{i}$ and $\alpha T_{H}$ is the parameter sets to be estimated. Here, $x_{i}$ is called FH components, which is denoted by two variables: time $t_{i}$ and $f_{i}$. In fact, $\mathrm{FH}$ parameters, such as hop during, hop timing and hop frequency are estimated by the parameter sets $\left\{t_{i}, f_{i}\right\}$.

\section{B. Time Frequency Analysis}

The WVD of a signal s ( $\mathrm{t}$ ) is defined as follows:

$$
W_{f}(t, s)=\int_{-\infty}^{\infty} s\left(t+\frac{\tau}{2}\right) s^{*}\left(t-\frac{\tau}{2}\right) e^{-j 2 \pi f \tau} d \tau
$$

Then, we can write the WVD of the FH signals $\mathrm{x}(\mathrm{t})$ as

$$
\begin{aligned}
W_{x}(t, f)= & \sum_{i=1}^{n} W_{x_{i}}(t, f)+2 \sum_{k=1}^{n-1} \sum_{=k+1}^{n} W_{x_{0}}\left(t-t_{m k l}, f-f_{m k l}\right) \times \cos \left[2 \pi f_{d k l}\left(t-t_{m k l}\right)\right. \\
& \left.-t_{d k l}\left(f-f_{m k l}\right)+2 \pi f_{d k l} t_{m k l}\right]
\end{aligned}
$$

where $\left(t_{d k l}, f_{d k l}\right)=\left(t_{k}-t_{l}, f_{k}-f_{l}\right)$ corresponds to the difference in the time and frequency shifts and $\left.\left(t_{m k l}, f_{m k l}\right)=\left(t_{k}+t_{l}\right) / 2,\left(f_{k}+f_{l}\right) / 2\right]$ is the average TF shifts between two components about FH signals. $W_{x_{0}}(t, f)$ is WVD of the arbitrary of "base” signal $x_{0}(t)$,

$$
W_{x_{0}}(t, f)=\left\{\begin{array}{lr}
\frac{1}{\pi\left(f-f_{0}\right)} \sin \left[4 \pi\left(f-f_{0}\right)\left(T_{H}-\mid t\right)\right] & |t|<T_{H} \\
0 & |t|>T_{H}
\end{array}\right.
$$

and $W_{x_{i}}(t, f)$ is the WVD of the component signal $x_{i}(t)$ as

$$
W_{x_{i}}(t, f)=\int_{-\infty}^{\infty} X_{i}\left(t+\frac{\tau}{2}\right) x_{i}^{*}\left(t-\frac{\tau}{2}\right) e^{-j 2 \pi f \tau} d \tau=W_{x_{i}}\left(t-t_{i}, f-f_{i}\right)
$$

In the above equation (5), the $n$ terms in the first sum corresponds to the auto-components $W_{x_{i}}(t, f)$, which it is clear that $W_{x_{i}}(t, f)$ is centered along the time $t_{i}$ and frequency $f_{i}$ for any component, whereas the last sets of terms are the cross terms, which are located at the average time $t_{m k l}$ and frequency $f_{m k l}$. It can be seen that the cross terms are oscillatory in nature and could be removed from TF plane by smoothing or filtering, for example, SPWVD [19]. Fig.1 (a) gives the maximum sequence plot of SPWVD which shows peak domains including seven hop components using difference operator. However, we can hardly find the location of these maximums due to the existence of the noise and the interference, for example in SNR -2dB, shown in fig.1 (b). Thus, the hop timing, hop during and hop frequency consequently is not estimated correctly as in previous literatures. 


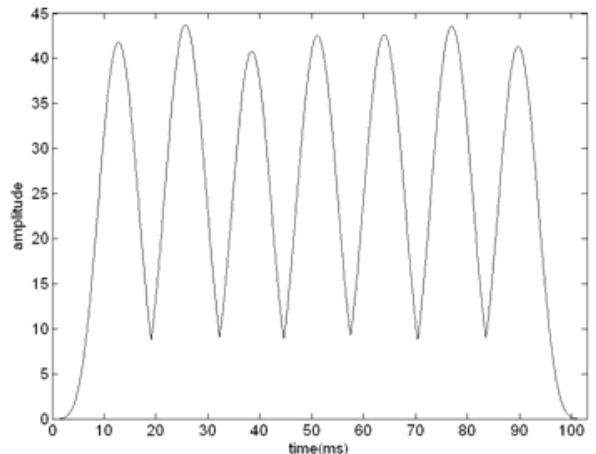

(a)

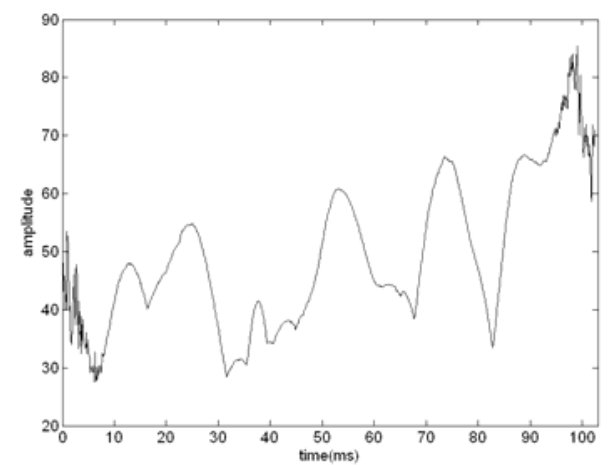

(b)

Fig. 1: The maximum sequence of SPWVD. (a) without noise. (b) $\mathrm{SNR}=-2 \mathrm{~dB}$

\section{C.Problem Formulation}

Our objective is to estimate hop instants and hop frequencies of the transmitted signals from noise signal $\mathrm{x}(\mathrm{t})$. From above analysis, the maximum locations on TF distribution represent the TF center of all components, which values is sensitive to noise. In this paper, we look peaks search problem upon as multi-modal function optimization and use MSPSO algorithm to locate the maximums so as to obtain the $\mathrm{TF}$ locations of all components which use to estimate parameters of $\mathrm{FH}$ signals.

The particle is a candidate solution of evaluating TF parameters, which is represented by a 2-dimensional realvalued vector, i.e. $\left(P_{i 1}, P_{i 2}\right)=(t, f)$.A certain number of particles form a species population and as particles moves through the search space, it compares its objective function with the best that it has ever attained so far. The behavior is repeated until all particles are gathered around the species seed of each species according to the distance measure, or a maximum iteration is encountered. With reference to (7), TF distribution (5) is chosen as the objective function in this study. The parameter estimation of signal (3) can be achieved by searching suitable $P_{s d}, d=1,2$ such that the objective function is maximized, i.e.

$$
\left(t^{*}, f^{*}\right)=\arg \min _{(t, f) \in S} W_{x}(t, f)
$$

where $\mathrm{S}$ is search space which admitted for subpopulations.

\section{EstimATION ALGORITHM}

Given a sequence of samples $\mathrm{x}(\mathrm{n})$ of the observed signal, with $n=0,1, \cdots N$ and $\mathrm{N}$ is the sample numbers, the estimation algorithm is based on the following steps:

1) Compute the discrete SPWVD of the signal $x(n)$.

2) Generate an initial random population with initial locations and velocities containing $\mathrm{H}$ particles chosen from the interval $[1, \mathrm{~N}]$. The initial personal best location is set by its current position.

3) Given species radius, each particle i moves around the search space, and changes its velocity according to (2) and position as (1b).

4) Find a new species seed s of assigning particle i to appropriate species s using MSPSO algorithm in (8). For a single FH signals, there are not overlap in time domain,so the distance measure is defined by the following equation

$$
d(i, s)=\left|x_{i 1}-x_{i 1}\right|
$$

5) If the pre-specified number of iterations is not achieved or not all particles are gathered, go back to steps 3.

6) Output the species seeds, i.e. time and frequency center of all obtained FH signal components.

In this algorithm, neither the number of species nor the population of each species is pre-determined [17], so 
the number and parameters of FH components are blind estimated.

\section{SIMULATION STUDIES}

The performance of the method has been evaluated by simulation in the presence of additive white Gaussian noise using the packet software of MATLAB 7.0. An example is useful to illustrate the estimating procedure. The hop frequency alphabet is $\{1.5 \mathrm{kHz}, 1.75 \mathrm{kHz}, 2.0$ kHz, $2.25 \mathrm{kHz}, 2.5 \mathrm{kHz}, 2.75 \mathrm{kHz}$, and $3.0 \mathrm{kHz}$. The sample rate is $10 \mathrm{kHz}$. The hop during is $12.8 \mathrm{~ms}$.

Figure 2 shows the evolution of clusters of a species in a particle swarm when the whole population is 500 , the maximum velocity is twenty percent of the variable range, species radius is 65 , and the maximum number of iteration is 300. The horizontal axis corresponds to time while the vertical one represents normalized frequency. During the evolution of population, each particle moves around the search space and immigrate from one species to another until the whole population is partitioned into different species, which the number of species is the number of signal components, i.e. 7. The trajectory of a particle can be guaranteed to converge if the parameters of PSO are chosen so that the corresponding particle's location always falls in the convergent region as shown in Fig.2 (ac). For the two acceleration coefficients and the inertia weight, we set the standard values $2.05,0.8$, respectively as in [18].

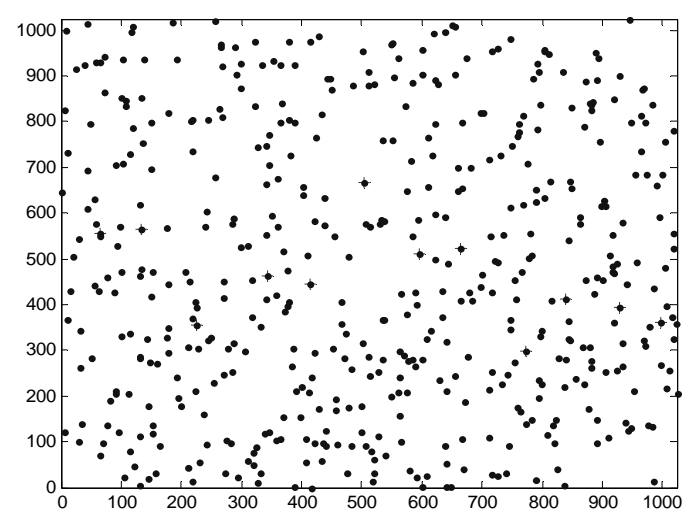

(a)

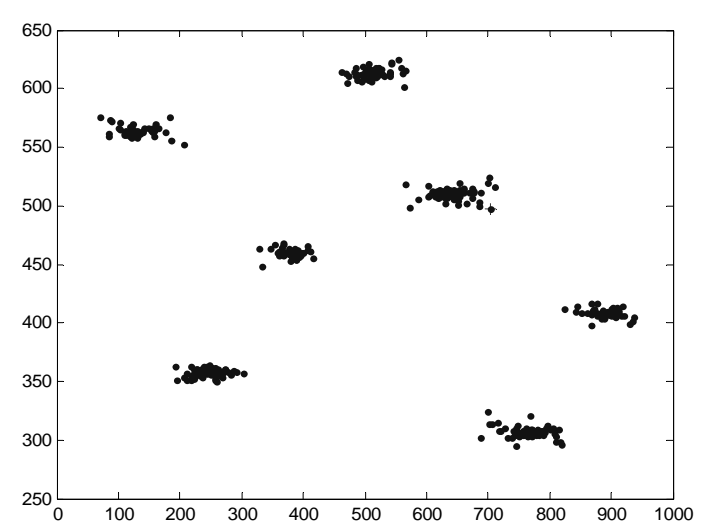

(b)

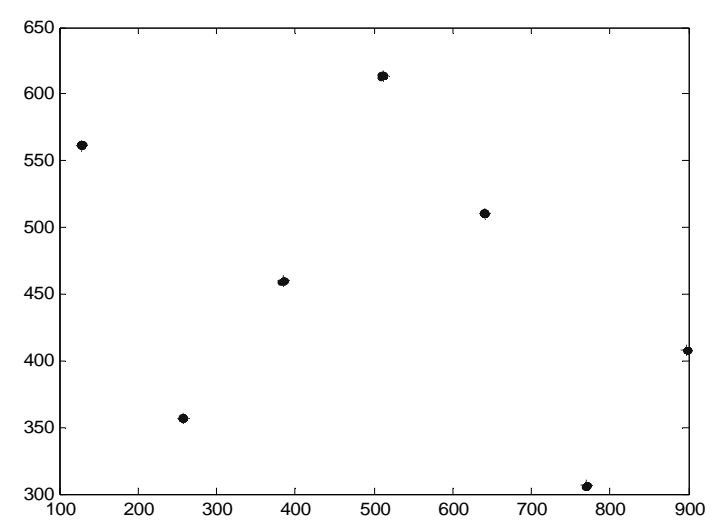

(c)

Fig.2: The evolution of the swarm population in TF plane. The dots indicate the locations of the particles, and the red crosses indicate the positions of component center. (a) iter $=0$. (b) iter $=10$. (c) iter $=50$.

The performance of this method has been evaluated by simulation in the presence of additive white Gaussian noise. The proposed algorithm was tested for 30 independent runs (trials). In each run, the same parameter as extracted above was used. Table 1 presents the time and frequency location of all the seven components in different SNR. The units in Table 1 are time or frequency sample. We can observe, as expected, the estimated parameter values are similar to the true one below $0 \mathrm{~dB}$, which the absolute error is less than 3 samples.

\begin{tabular}{ccc}
\multicolumn{3}{c}{ TABLE I } \\
THE ESTIMATED PARAMETER VALUES OF FH COMPONENTS \\
\cline { 2 - 4 } $\begin{array}{c}\text { Component } \\
\text { index }\end{array}$ & \multicolumn{2}{c}{ SNR=-2dB } \\
\cline { 2 - 3 } & time & frequency \\
\hline$\# 1$ & 128.2616 & 562.1197 \\
$\# 2$ & 256.2342 & 358.0306 \\
$\# 3$ & 381.0979 & 460.2252 \\
$\# 4$ & 517.2211 & 611.8515 \\
$\# 5$ & 645.4963 & 511.8684 \\
$\# 6$ & 765.3898 & 308.1621 \\
$\# 7$ & 898.6748 & 408.1488 \\
\hline
\end{tabular}


TABLE II.

THE ESTIMATED PARAMETER VALUES OF FH COMPONENTS

\begin{tabular}{ccc}
\hline \multirow{2}{*}{\begin{tabular}{c} 
Component $\begin{array}{c}\text { SNdex } \\
\text { inde=0dB }\end{array}$ \\
\cline { 2 - 3 }
\end{tabular}} & time & frequency \\
\hline$\# 1$ & 127.5250 & 562.3153 \\
$\# 2$ & 256.0044 & 357.8336 \\
$\# 3$ & 382.2852 & 460.0340 \\
$\# 4$ & 515.2323 & 612.9383 \\
$\# 5$ & 643.4909 & 511.3633 \\
$\# 6$ & 766.4841 & 307.2144 \\
$\# 7$ & 899.0985 & 408.4468 \\
\hline
\end{tabular}

TABLE III.

THE ESTIMATED PARAMETER VALUES OF FH COMPONENTS

\begin{tabular}{ccc}
\hline \multirow{2}{*}{$\begin{array}{c}\text { Component } \\
\text { index }\end{array}$} & \multicolumn{2}{c}{ SNR=2dB } \\
\cline { 2 - 3 } & time & frequency \\
\hline$\# 1$ & 128.0765 & 561.7338 \\
$\# 2$ & 256.6829 & 357.2694 \\
$\# 3$ & 385.0712 & 460.2256 \\
$\# 4$ & 511.4474 & 613.7101 \\
$\# 5$ & 640.4656 & 510.8083 \\
$\# 6$ & 770.2921 & 306.3655 \\
$\# 7$ & 897.5453 & 407.5886 \\
\hline
\end{tabular}

A simplified explanation of the above algorithm is designed for hop during estimation, which is obtained by computing the average for the difference of all the seeds taking account of time variable. The mean -square error of this parameter estimation at different SNR values in the range of $-10 \mathrm{~dB}$ to $2 \mathrm{~dB}$ is shown in Fig. 3 using the proposed method and the algorithm in [7]. It can be seen from Fig. 3 that the modified MSPSO algorithm using our approach can be used to estimate parameter of FH signals This algorithm achieves several goals comparing with [9] First, optimization search approach is reliable, i.e. no mutations of performance, in a wider range of SNR, since there is not a threshold effect, although lower variance is obtained using SPWVD above the SNR threshold. A second benefit of this approach is that there is no error propagation phenomenon because time and frequency variables are independent. Finally, another benefit of our method is the ability to estimation parameter correctly in the very low SNR noise, which is in accordance with component location estimation formerly.

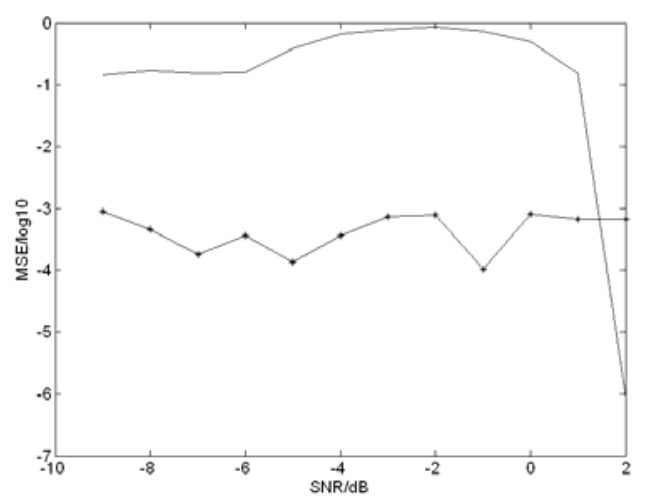

Fig.3 hop during estimation variance

\section{CONCLUSIONS}

By applying the proposed PSO optimization for TF distribution, blind parameter estimation of frequency hopping signal is solved taking account of the relation between peaks location on the TF plane and component centers of frequency hopping signals. Differently from other known methods, the proposed method provides a direct search method of time and frequency parameter of FH signals. The experimental results proved that the theoretical derivation is correct and the proposed method is effective and feasible under the low SNR. One future work will include how to unify TF representation and PSO having simple and robust properties, which could give high-precision.

\section{ACKNOWLEDGMENT}

This paper is based upon supported by Natural Science Foundation of Henan Province Educational Committee under Grants No.2011A510021, Scientific Research Foundation for High-level talent, Xinyang Normal University.

\section{ACKNOWLEDGMENT}

[1] J. J. Lehtomaki, M. Juntti, "Detection of frequency hopping signals using a sweeping channelized radiometer,” Dignal Processing, Vol. 85, pp. 2030-2043, 2005.

[2] J. J. Lehtomaki, "Maximum based detection of slow frequency hopping signals,” IEEE Communications Leters, vol. 7, No.5, pp. 201-203, 2003. 
[3] G. Lopez, O. Y. Ojeda, “Atomic decomposition based complex radar interception,” IEE Proceedings Radar, Sonar and Navigation, pp.323-331, Aug, 2003.

[4] S. Mallat and Z. Zhang, "Matching pursuits with timefrequency dictionaries,” IEEE Trans. Signal Processing, vol. 41, pp. 3397-3415, Dec.1993.

[5] Ye Yuan, Wen-bo Mei, Si-liang Wu, "Hop Period Estimation for Frequency Hopping Signals Based on Hilbert-Huang Transform,” 2008 Congress on Image and Signal Processing, vol.5, pp.452-455, 2008.

[6] Jiantao Guo, "Adaptive time-frequency parameterization of frequency-hopping signals based on evolutionary algorithm,” 2010 Third International Joint Conference on Computer Science and Optimization, vol.1, pp.279-282, 2010.

[7] S. Barbarossa, "Parameter estimation of spread spectrum frequency hopping signals using time-frequency distributions,” First IEEE Signal Processing Working on Signal Processing Advances in Wireless Communications, pp. 213-216, 1997.

[8] Zhang Chao-yang, Cao Qian-qian, and Chen Wen-zheng, "Bling separation and parameter estimation of multiple frequency-hoppings," Journal of Zhejiang University (Engineering Science), Vol.39, No.4, pp. 465-470, 2005 (in Chinese).

[9] Zhao Jun, Zhang Zhaoyang, Lai Lifeng, "Blind parameter estimation of frequency-hopping signals based on timefrequency analysis,” Journal of circuits and systems, Vol.8, No.3, pp.213-216, 2003 (in Chinese).

[10] F.Auger, P. Flandrin, "Improving the readability of time frequency and time-scale representations by the reassignment method”, IEEE Trans. Signal Processing, Vol.43, No.5, pp.1068-1089, 1995.

[11] Tian Yan, Gou Yanxin, Chi Wanhong, Bu Weidong, "Reassignment-based frequency hopping signal timefrequency analysis," Communication Countermeasures, vol.96, No. 1, pp:8-12, 2007.

[12] Xu Mankun, Ping Xijian, Li Tianyun, Xu Mantian, “A new time-frequency spectrogram analysis of $\mathrm{FH}$ signals by image enhancement and mathematical morphology," Fourth International Conference on Image and Graphics, ICIG 2007, pp.610-615, 2007.

[13] J.Kennedy, R C Eberhart, "Particle Swarm optimization," in proceedings of IEEE international conference on neural networks, Picataway, NJ, pp.1942-1948, 1993.

[14] K. E. Parsopoulos, M. N. Vrahatis, "UPSO: A Unified Particle Swarm Optimization Scheme,” Lecture Series on Comp. and Computational Sci., Vol. 1, Proc. of the Int. Conf. of Computational Methods in Sci. and Eng., pp. 868873, 2004.

[15] J.-P. Li, M.E. Balazs, G.T. Parks, P.J. Clarkson, “A Genetic Algorithm using Species Conservationfor Multimodal Function Optimization,” Journal of Evolutionary Computation,vol. 10, no. 3, pp. 207-234, 2003.

[16] R. Brits, A.P. Engelbrecht, F. van den Bergh, "A niching particle swarm optimizer," Proc. 4thAsia-Pacific Conf. on Simulated Evolution and Learning, vol. 2, pp. 692-696, 2002.

[17] M Iwamatsu, "Multi-species particle swarm optimizer for multimodal function optimization", IEICE Trans. INF.\&SYST., Vol.E89-D, No.3, pp.1181-1188, 2006.

[18] Masao Iwamatsu, "Locating All the Global Minima Using Multi-species Particle Swarm optimizer the inertia weight and the constriction factor variants," in IEEE Congress on Evolutionary Computation, pp. 16-21, 2006.
[19] K Shubha, G.B. Bartels, “A comparison of the existence of "cross terms" in the Wigner Distribution and the squared magnitude of the wavelet transform and the short time foourier transform," IEEE Transaction on Signal Processing, vol.40, No.10, pp.2498-2517, 1992.

Guo Jiantao received the M.Sc. degree in signal and information processing (optical information encryption) from DaLian University of Science and Technology, Dalian, China and the Ph.D. degree in signal and information processing (time frequency analysis) from Huazhong University of Science and Technology, Wuhan, China, in 2004 and 2009, respectively.

$\mathrm{He}$ is an associate professor with the College of Physics and Electronic Engineering, Xinyang Normal University, Xinyang, China. He maintains an active interest in the field of time frequency analysis and swarm intelligence. Further research interests include spread spectrum signal processing, digital watermarking, and blind source separation.

Wang Lin received the B.Sc. degree from the Yunnan University, Kunming, China, in 1985 and subsequently worked in the College of Physics and Electronic Engineering, Xinyang Normal University, Xinyang, China. Her research focuses on frequency-hopping signal processing and evolutionary computation. 